\title{
The coupled electronic oscillators vs the sum-over-states pictures for the optical response of octatetraene
}

\author{
Guanhua Chen and Shaul Mukamel \\ Department of Chemistry, University of Rochester, Rochester, New York 14627 \\ David Beljonne and Jean-Luc Brédas \\ Service de Chimie des Matériaux Nouveaux, Centre de Recherche en Electronique et Photonique \\ Moléculaires, Université de Mons-Hainaut, Place du Parc 20, 7000 Mons, Belgium
}

(Received 8 November 1995; accepted 29 December 1995)

\begin{abstract}
A coupled electronic oscillator (CEO) analysis of the third harmonic generation (THG) spectrum for octatetraene is presented. The dominant oscillators and their couplings are identified using tree diagrams. The correspondence between the dominant oscillators in the CEO picture and the relevant excited states in the sum-over-states (SOS) description is demonstrated. The important channels in the SOS are related to the dominant oscillator pathways in the CEO picture. (C) 1996 American Institute of Physics. [S0021-9606(96)01613-3]
\end{abstract}

\section{INTRODUCTION}

In the coupled electronic oscillator (CEO) picture, a many-electron system is mapped onto a collection of coupled normal modes representing the electron-hole pairs. ${ }^{1-3}$ The dynamics of electronic excitations can then be calculated by following the evolution of these oscillators. This method eliminates the need for calculating the global ground and excited state wave functions, and provides an efficient algorithm for evaluating linear and nonlinear response of an electronic system subject to external electromagnetic fields. It also provides an intuitive picture for underlying complex electronic dynamics. This technique has been applied successfully to the investigation of nonlinear optical properties of conjugated polyenes. ${ }^{1-6}$ On the other hand, the sum-overstates (SOS) method is commonly used in quantum chemical calculations of the optical susceptibilities. ${ }^{7-11}$ It is derived by a perturbative expansion of the wave function in powers of the applied field ${ }^{7}$ and requires the calculations of both the ground state and excited states wave functions and the transition dipole moments between them. Although it is relatively computationally expensive, it provides detailed information about the system and helps develop physical intuition. It would be interesting to compare the CEO picture and the SOS formalism.

Tree diagrams, which illustrate the couplings among the oscillators and the origin of high order electronic response to the external fields, were introduced recently. ${ }^{5}$ These diagrams were employed to analyze the contributions of various oscillators to the off-resonant polarizabilities of octatetraene. The dominant oscillators were projected onto the HartreeFock oscillator (HFO) representation (a Liouville space whose basis vectors are the oscillators representing two Hartree-Fock molecular orbitals). This projection provides a connection between the CEO picture and the SOS method. However, a comparison between these two methods over a wide range of frequencies is lacking. This comparison is needed for establishing the correspondence of the two methods and for further development of the CEO picture.

In this report, we present a thorough study of nonlinear optical processes of octatetraene using the CEO picture. Although the CEO is expected to work better for larger systems with more electrons, where the excitations are collective, octatetraene is chosen for several reasons. It is centrosymmetric and thus both the oscillators and the states can be categorized according to their symmetries. This leads to a simplification of the description. Detailed highly resolved spectra are available $^{12}$ and a preliminary study has already been done. ${ }^{5}$ Here, we will calculate the dispersed third-harmonicgeneration (THG) spectrum, identify the dominant oscillators of different orders at various frequencies, and investigate the nature of these oscillators. We will then compare these results to the description offered by the SOS scheme, with a special emphasis on the connection between the dominant oscillators and the essential excited states. We will attempt to establish the similarities and identify the differences between the two pictures. Some work has been carried out, which sheds light upon the connection between the CEO and SOS descriptions of asymmetric molecules. ${ }^{3}$ A complete study of another important organic nonlinear optical compound, dimethyl-amino-nitro-stilbene (DANS), will be published elsewhere. ${ }^{13}$

\section{THE COUPLED-OSCILLATORS AND THE SUM- OVER-STATES FORMALISMS}

An $N$-electron system with $N$ electronic orbitals is mapped into a collection of $N(N+1) / 2$ oscillators with frequencies $\Omega_{\nu}$. These include $N^{2} / 4$ particle-hole oscillators, $N(N+2) / 8$ particle-particle oscillators, and $N(N+2) / 8$ hole-hole oscillators. Among the particle-particle and holehole oscillators there are $N$ zero frequency oscillators. Each oscillator $\nu$ has a coordinate $\hat{Q}_{\nu}$ and a momentum $\hat{P}_{\nu}$ which are the natural collective coordinates of the system. By expanding the density matrix $\rho_{n m}(t)$ in these coordinates we obtain $\delta \rho_{n m}(t) \equiv \rho_{n m}(t)-\rho_{n m}^{(0)}=\Sigma_{\nu}\left[Q_{\nu}(t) \hat{Q}_{\nu}+P_{\nu}(t) \hat{P}_{\nu}\right]$, where $\rho^{(0)}$ is the reduced Hartree-Fock ground state density matrix, and $Q_{\nu}(t)$ and $P_{\nu}(t)$ are time dependent coefficients. ${ }^{3}$ 
The $n$th order polarization is calculated using the following expression: ${ }^{3}$

$$
P^{(n)}=\sum_{\nu} d_{\nu} Q_{\nu}^{(n)},
$$

where $Q_{\nu}^{(n)}$ represents the amplitude of the $\nu$ th electronic oscillator to $n$th order in the external field and $d_{\nu}$ is its dipole moment,

$$
d_{\nu}=-\sum_{m} 2 \sqrt{2} e z(m) \mathscr{C}_{m m, \nu}^{-1}
$$

where $z(m)$ is the coordinate of $m$ th atom in the chain $(z)$ direction and $\mathscr{U}_{m n, \nu}^{-1}$ is the $m n$ component of oscillator $\nu$. The $n$th order polarizability $\chi_{n}$ is given $b^{3}$

$$
\chi_{n}=\sum_{\nu} d_{\nu} Q_{\nu}^{(n)} / \mathscr{E}^{n}
$$

where $\chi_{1}=\alpha, \chi_{2}=\beta, \chi_{3}=\gamma$, and $\mathscr{E}$ is the external field. To distinguish the contributions from different oscillators to the polarizabilities, we introduced the following quantities: ${ }^{3}$ $\alpha_{\nu}=d_{\nu} Q_{\nu}^{(1)} / \mathscr{E}, \beta_{\nu}=d_{\nu} Q_{\nu}^{(2)} / \mathscr{C}^{2}$, and $\gamma_{\nu}=d_{\nu} Q_{\nu}^{(3)} / \mathscr{E}^{3}$. Thus, $\alpha=\Sigma_{\nu} \alpha_{\nu}, \beta=\Sigma_{\nu} \beta_{\nu}$, and $\gamma=\Sigma_{\nu} \gamma_{\nu}$.

There are usually only a few oscillators which dominate the optical susceptibilities. ${ }^{3,5}$ These can be identified using tree diagrams. ${ }^{5}$ The interaction between the ground state and the external field leads to first-order oscillators. The firstorder oscillators then couple with the external field and among themselves to produce the second-order oscillators; finally the second order oscillators couple with the external field and with the first-order oscillators to produce the thirdorder oscillators. In the tree diagrams, we use circles, squares and triangles to represent the dominant oscillators of first-, second-, and third-order, respectively, and lines to represent the nonlinear couplings between the oscillators or with the external field. These circles, squares, triangles, and lines constitute the pathways representing the nonlinear optical processes in the system. Since some dominant oscillators have small or zero dipole moments, the related pathways do not contribute much to the polarizability at a given order, although these pathways may be vital to the creation of nextorder optical processes.

In the SOS method, the optical properties are related to the eigenvalues and dipole matrix elements of the global many-electron eigenstates, and physical intuition is developed through the properties of the ground and excited states. ${ }^{7-11,14-17}$ For instance, the third order static polarizability can be written as

$$
\begin{aligned}
\gamma \propto & -\sum_{e} \frac{\mu_{g e}^{4}}{E_{g e}^{3}}+\sum_{e} \sum_{e^{\prime}} \frac{\mu_{g e}^{2} \mu_{e e^{\prime}}^{2}}{E_{g e}^{2} E_{g e^{\prime}}} \\
& +\sum_{e} \frac{\mu_{g e}^{2}\left(\mu_{e e}-\mu_{g g}\right)^{2}}{E_{g e}^{3}} \\
& +\sum_{e} \sum_{e^{\prime}} \sum_{e^{\prime \prime}} \frac{\mu_{g e} \mu_{e e^{\prime}} \mu_{e^{\prime} e^{\prime \prime}} \mu_{e^{\prime \prime} g}}{E_{g e} E_{g e^{\prime}} E_{g e^{\prime \prime}}},
\end{aligned}
$$

where, $\mu_{g e}$ or $\mu_{e^{\prime \prime} g}$ is the transition dipole moment between the ground $(g)$ and an excited ( $e$ or $\left.e^{\prime \prime}\right)$ state. $\mu_{e e^{\prime}}$ or $\mu_{e^{\prime} e^{\prime \prime}}$ is the transition dipole moment between two excited states $(e$ and $e^{\prime}$ or $e^{\prime}$ and $\left.e^{\prime \prime}\right) . \mu_{g g}\left(\mu_{e e}\right)$ is the static dipole moment of the ground (excited) state. $E_{g e}$ or $E_{g e^{\prime}}$ or $E_{g e^{\prime \prime}}$ is the energy difference between the ground $(g)$ and an excited ( $e$ or $e^{\prime}$ or $\left.e^{\prime \prime}\right)$ states. Usually only a handful of "essential states" are important. For conjugated polyenes, a three-essential-state or four-essential-state model ${ }^{12,17}$ has been proposed to account for the nonlinear optical spectra. The transitions among these essential states form important channels. These channels provide physical intuition about the underlying linear and nonlinear optical properties.

There are many relationships between these two formalisms. $d_{\nu}$ in Eq. (2) may be expressed as a linear combination of the transition dipole moments between Hartree-Fock molecular orbitals or the static dipole moments of Hartree-Fock molecular orbitals. $\mu_{g g}, \mu_{e e}, \mu_{g e}, \mu_{e^{\prime \prime} g}, \mu_{e e^{\prime}}$, and $\mu_{e^{\prime} e^{\prime \prime}}$ in Eq. (4) can be expressed in terms of linear combination of the transition dipole moments and static dipole moments of Hartree-Fock molecular orbitals as well. In the time dependent Hartree-Fock (TDHF) or random phase approximation (RPA) (Refs. 18-20) the frequencies $\Omega_{\nu}$ of the oscillators which constitute only of the particle-hole components are exactly the energy differences between the excited states and ground state, and thus corresponds to $E_{g e}$ or $E_{g e^{\prime}}$ or $E_{g e^{\prime \prime}}$ in Eq. (4). Since different approximations are usually employed in both calculations, the calculated polarizabilities are not exactly the same. To connect these two descriptions, we may project the oscillators onto the HFO representation, which is made of the oscillators between two Hartree-Fock molecular orbitals, ${ }^{3}$ and expand the excited states in terms of the Hartree-Fock molecular orbitals.

\section{RESULTS}

\section{A. Electronic oscillators and excited states}

The model used in the CEO calculations is the PariserParr-Pople (PPP) Hamiltonian. ${ }^{3}$ The parameters of the model employed in the calculations are chosen to fit the chemical structure (the bond-length-alternation is $0.09 \AA$ ) and the absorption frequency $(\sim 4 \mathrm{eV})$ of octatetraene. ${ }^{21}$ This corresponds to $a_{0}=1.2935 \AA, U_{0}=11.13 \mathrm{eV}, \bar{\beta}=-2.4 \mathrm{eV}$, $\beta^{\prime}=-5.6 \mathrm{eV} \AA^{-1}, K=80 \mathrm{eV} \AA^{-2}, \bar{x}=1.512 \AA$, and $\epsilon=1.5$. These values are slightly different from those in Ref. 3 .

For octatetraene $(N=8)$, there are 36 oscillators. In Table I, we list the frequencies $\Omega_{\nu}$, dipole moments $d_{\nu}$, and oscillator strengths $f_{\nu}$ of these oscillators. Since octatetraene is centrosymmetric, the oscillators are divided into the gerade $(g)$ oscillators $\left(A_{g}\right.$ and $\left.A_{g}^{\prime}\right)$ and the ungerade $(u)$ oscillators $\left(B_{u}\right.$ and $\left.B_{u}^{\prime}\right)$ according to their inversion symmetry. ${ }^{22}$ For $A_{g}$ and $A_{g}^{\prime}$ oscillators, both $d_{\nu}$ and $f_{\nu}$ are zero. There are $8 A_{g}$ and $8 B_{u}$ oscillators which are made of purely particle-hole pairs and they may cause resonances at their oscillator frequencies. The other $12 A_{g}^{\prime}$ and $8 B_{u}^{\prime}$ oscillators consist of particle-particle or hole-hole components. These oscillators contribute to high order polarizabilities (not $\alpha$ ) but do not show up as resonances in optical spectra. Among these 20 
TABLE I. Properties of the harmonic oscillators. ${ }^{\mathrm{a}}$

\begin{tabular}{|c|c|c|c|c|c|c|c|c|}
\hline$A_{g}$ & (1) & (2) & (3) & (4) & (5) & (6) & (7) & (8) \\
\hline$\Omega_{\nu}^{\delta}$ & 5.764 & 5.857 & 7.827 & 7.921 & 9.133 & 9.875 & 11.350 & 12.836 \\
\hline$A_{g}^{\prime}$ & (1) & (2) & (3) & (4) & (5) & (6) & (7) & (8) \\
\hline$\Omega_{\nu}$ & 0 & 0 & 0 & 0 & 0 & 0 & 0 & 0 \\
\hline$A_{g}^{\prime}$ & (9) & (10) & (11) & (12) & & & & \\
\hline$\Omega_{\nu}$ & 2.765 & 2.765 & 3.702 & 3.702 & & & & \\
\hline$B_{u}$ & (1) & (2) & (3) & (4) & (5) & (6) & (7) & (8) \\
\hline$\Omega_{\nu}$ & 3.916 & 7.073 & 7.181 & 8.073 & 9.532 & 10.705 & 11.021 & 13.056 \\
\hline$d_{\nu}$ & 4.316 & 0.848 & 0 & 0.397 & 0.214 & 0 & 0.139 & 0.027 \\
\hline$f_{\nu}$ & 7.297 & 0.513 & 0 & 0.127 & 0.042 & 0 & 0.021 & 0.0 \\
\hline$B_{u}^{\prime}$ & (1) & (2) & (3) & (4) & (5) & (6) & (7) & (8) \\
\hline$\Omega_{\nu}$ & 1.081 & 1.081 & 1.684 & 1.684 & 2.019 & 2.019 & 4.783 & 4.783 \\
\hline$d_{\nu}$ & 3.487 & 3.487 & 4.013 & 4.013 & 4.128 & 4.128 & 0.093 & 0.093 \\
\hline$f_{\nu}$ & 0 & 0 & 0 & 0 & 0 & 0 & 0 & 0 \\
\hline
\end{tabular}

a For $A_{g}$ and $A_{g}^{\prime}$ oscillator, $d_{\nu}=0$ and $f_{\nu}=0, \Omega_{\nu}$ is in the units of eV, $m_{0}=3.019 m_{e}\left(m_{e}\right.$ is the mass of an electron) and $d_{\nu}$ is in the units of $e \AA$ (which is 4.8032 D). The values of $d_{\nu}$ in Table I of Ref. 5 should be multiplied by $\sqrt{2}$.

oscillators, 8 have zero frequency and are of $A_{g}$ symmetry. We will employ the following notation: $A_{g}(n), A_{g}^{\prime}(n)$, $B_{u}(n)$, and $B_{u}^{\prime}(n)$ will denote the $n$th oscillator in each category.

In the SOS formalism, the singlet excited states were calculated by employing the intermediate neglect of differential overlap (INDO) Hamiltonian ${ }^{23}$ and using the multireference double configuration interaction (MRD-CI) technique. ${ }^{24}$ The geometry was optimized at the HartreeFock semiempirical Austin Model 1 (AM1) (Ref. 25) level. We took the lowest 40 singlet excited states and plugged them in the SOS expressions to obtain the values of linear and nonlinear polarizabilities.

We will denote by $n A g$ the $n$th $A g$ state and $n B u$ the $n$th $B u$ state in order of increasing energy.

\section{B. Absorption and THG spectra}

In Fig. 1 we compare the absorption spectrum of octatetraene using both the CEO picture and the SOS method. The frequency $\omega$ is scaled by the optical gap $\omega_{0}$ (which is $\Delta_{\mathrm{CEO}}=3.92 \mathrm{eV}$ for the $\mathrm{CEO}$ and $\Delta_{\mathrm{SOS}}=4.93 \mathrm{eV}$ for the SOS). Both the SOS and CEO spectra show a large absorption peak, $1 A$ and $1 a$, respectively. This peak carries almost all the oscillator strength. $1 A$ and $1 a$ are one-photon resonances corresponding to the $1 B u$ excited state and $B_{u}(1)$ oscillator, respectively.

In Fig. 2 we compare the THG spectrum of octatetraene for both the CEO and SOS pictures. The SOS spectrum has eight peaks. $3 A, 3 B^{\prime \prime}, 3 B^{\prime}, 3 B$, and $3 C$ are three-photon resonances caused by the $1 B u, 5 B u, 6 B u, 10 B u$, and $12 B u$ excited states, respectively. $3 A^{\prime}$ and $3 D$ are two-photon resonances caused by the $2 \mathrm{Ag}$ and $6 \mathrm{Ag}$ excited states, respectively. The CEO spectrum shows five peaks. The resonances in the intermediate region between $3 a$ and $3 b$ are too weak to be resolved. $3 a, 3 b, 3 c$, and $3 e$ are three-photon resonances corresponding to the $B_{u}(1), B_{u}(3), B_{u}(4)$, and $B_{u}(5)$ electronic oscillators, respectively. $3 d$ is a two-photon resonance due to $A_{g}(2)$. The dominant features in both spec- tra $\left(3 A, 3 B^{\prime} / 3 B, 3 C\right.$, and $\left.3 D\right)$ and $(3 a, 3 b, 3 c$, and $3 d)$ agree although $(3 C, 3 D)$ is blue shifted $\left(\sim 0.05 \omega_{0}\right)$ with respect to $(3 c, 3 d)$.

\section{Analysis and comparison}

We explored the connection between the CEO and SOS pictures for three frequencies; (1) the static limit $(\omega \rightarrow 0)$; (2) the $3 A / 3 a$ resonance; and (3) the $(3 C, 3 D) /(3 c, 3 d)$ resonance.

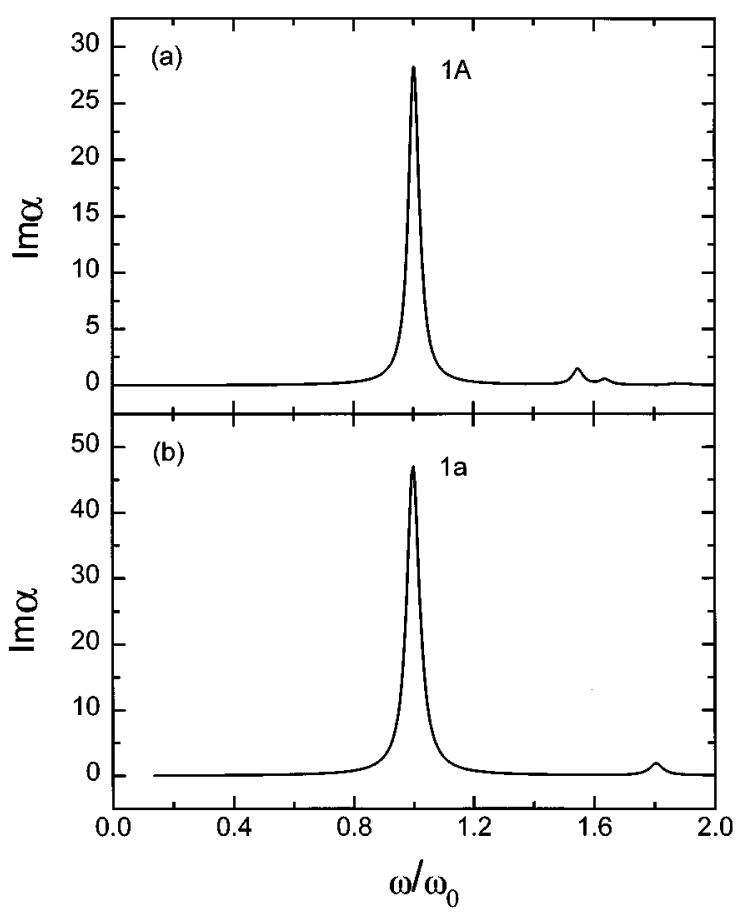

FIG. 1. Absorption spectrum for octatetraene. $\operatorname{Im}[\alpha(\omega)]$ is in units of $e \AA \mathrm{V}^{-1}$ (which is $1.441 \times 10^{-23} \mathrm{esu}$ ). (a) INDO/MRD-CI/SOS result with the optical gap $\omega_{0}=\Delta_{\mathrm{SOS}}=4.93 \mathrm{eV}$, and (b) PPP/CEO result with $\omega_{0}=\Delta_{\mathrm{CEO}}=3.92 \mathrm{eV}$. 


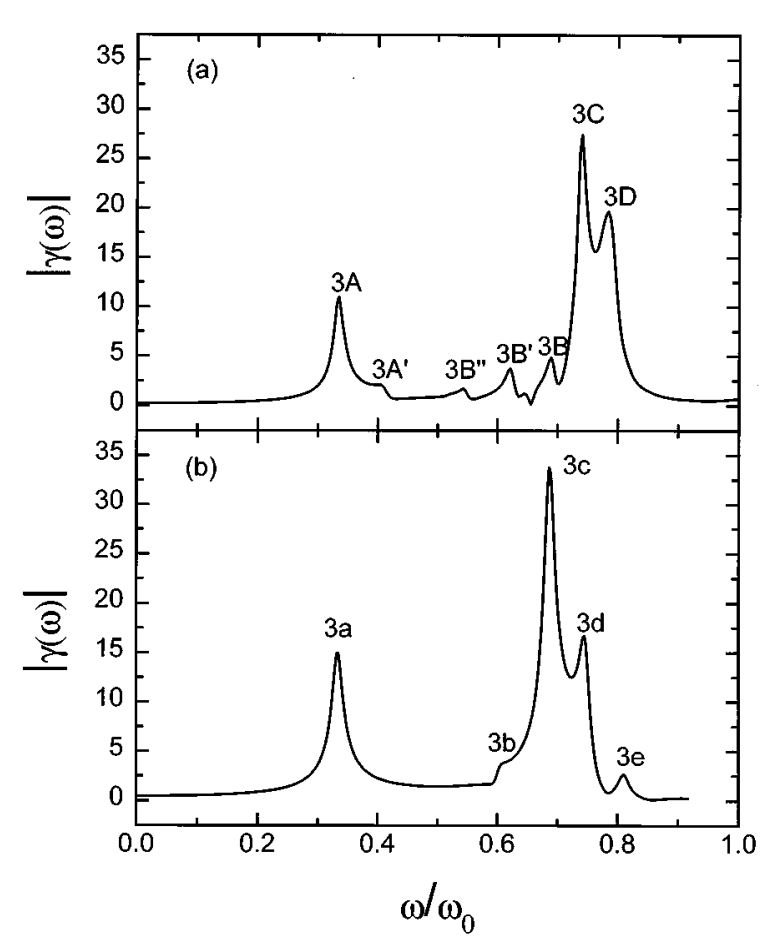

FIG. 2. THG spectrum for octatetraene. $|\gamma(\omega)|$ is in units of $e \AA^{4} \mathrm{~V}^{-3}$ (which is $1.297 \times 10^{-34} \mathrm{esu}$ ); (a) INDO/MRD-CI/SOS spectrum; and (b) PPP/CEO spectrum.

\section{The static limit}

In Fig. 3(a), we plot the contribution of oscillator $\nu$ to the linear polarizability $\alpha_{\nu}$ vs the oscillator frequency $\Omega_{\nu}$ for

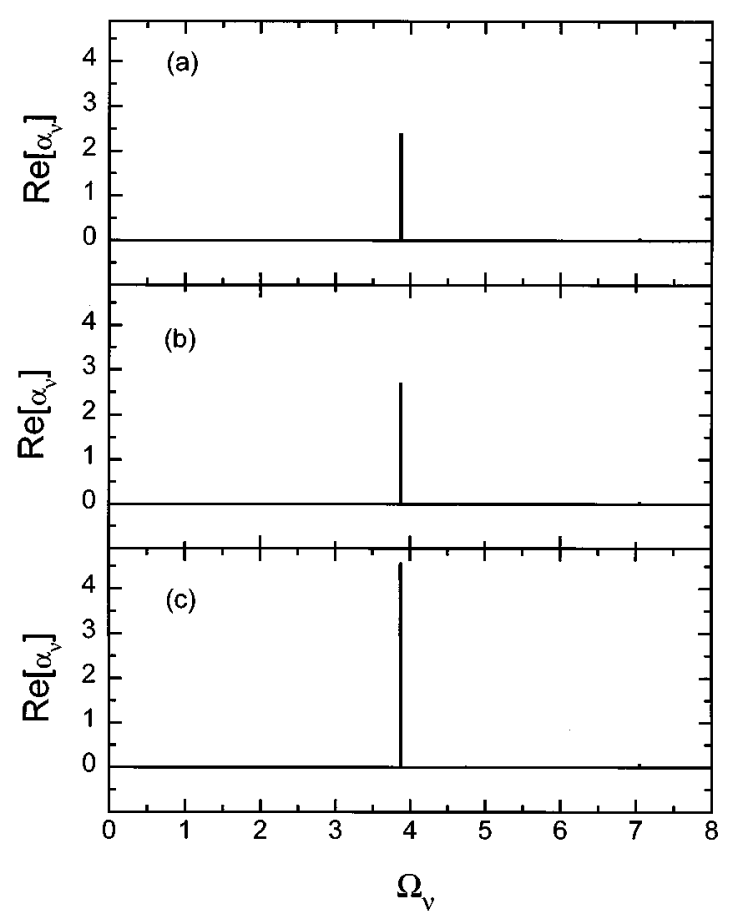

FIG. 3. $\operatorname{Re}\left[\alpha_{\nu}\right]\left(e \AA^{2} / \mathrm{V}\right)$ vs $\Omega_{\nu}$ (eV) for the PPP/CEO; (a) $\omega=0.1 \mathrm{eV}$; (b) 1.3 $\mathrm{eV}$, and (c) $2.7 \mathrm{eV}$.
TABLE II. The $\alpha_{\nu}$ of the $B_{u}(1)$ oscillator at different frequencies. ${ }^{\text {a }}$

\begin{tabular}{cccc}
\hline \hline$\omega(\mathrm{eV})$ & 0.1 & 1.3 & 2.7 \\
\hline$B_{u}(1)$ & $(2.407,0.003)$ & $(2.702,0.051)$ & $(4.562,0.306)$ \\
Total $^{\mathrm{b}}$ & $(2.472,0.003)$ & $(2.769,0.052)$ & $(4.637,0.307)$ \\
\hline \hline
\end{tabular}

${ }^{\mathrm{a}} \alpha_{\nu}$ is in the units of $e \AA^{2} / \mathrm{V}$.

${ }^{\mathrm{b}}$ Total: the sum of $\alpha_{\nu}$ from all $B_{u}$ oscillators.

an off-resonant low frequency $\omega=0.1 \mathrm{eV}$ in the CEO. Clearly there is only one dominant oscillator $B_{u}(1)$ whose contribution to linear susceptibility $\alpha_{\nu}=(2.407,0.003)$ $e \AA^{2} / \mathrm{V}$, as compared to the total $\alpha=(2.472,0.003) e \AA^{2} / \mathrm{V}$ (see Table II). The first number in a parenthesis is the real part of a complex number and the second number is the imaginary part. We will use this notation for $\alpha, \gamma$, and $Q_{\nu}$ in the rest of paper. This oscillator carries $7.297 / 8=91.2 \%$ of the oscillator strength, see Table I. It couples with the external field and with itself producing three second-order dominant oscillators $A_{g}^{\prime}(1), A_{g}^{\prime}(2)$, and $A_{g}(2)$ (Fig. 4). In third order (Fig. 5), the second order dominant oscillators $A_{g}^{\prime}(1)$, $A_{g}^{\prime}(2)$ and $A_{g}(2)$ couple with the external field and the firstorder oscillators $B_{u}(1)$ to produce the third-order oscillators $B_{u}^{\prime}(5), B_{u}^{\prime}(6), B_{u}(1)$, and $B_{u}(4)$. These coupling pathways have been depicted in the tree diagrams of Figs. 4 and 5 . Each of these pathways contributes to the $Q_{\nu}^{(2)}$ of $A_{g}(2)$ or $A_{g}^{\prime}(1)$ or $A_{g}^{\prime}(2)$ in second order and the $Q_{\nu}^{(3)}$ of $B_{u}(1), B_{u}(4)$, $B_{u}^{\prime}(5)$ or $B_{u}^{\prime}(6)$ in third order. These contributions to $Q_{\nu}^{(2)}$ and $Q_{\nu}^{(3)}$ are listed along the lines in Figs. 4 and 5. Since $A_{g}^{\prime}(1), A_{g}^{\prime}(2), B_{u}^{\prime}(5)$, and $B_{u}^{\prime}(6)$ contain the particleparticle and/or hole-hole components, they do not induce
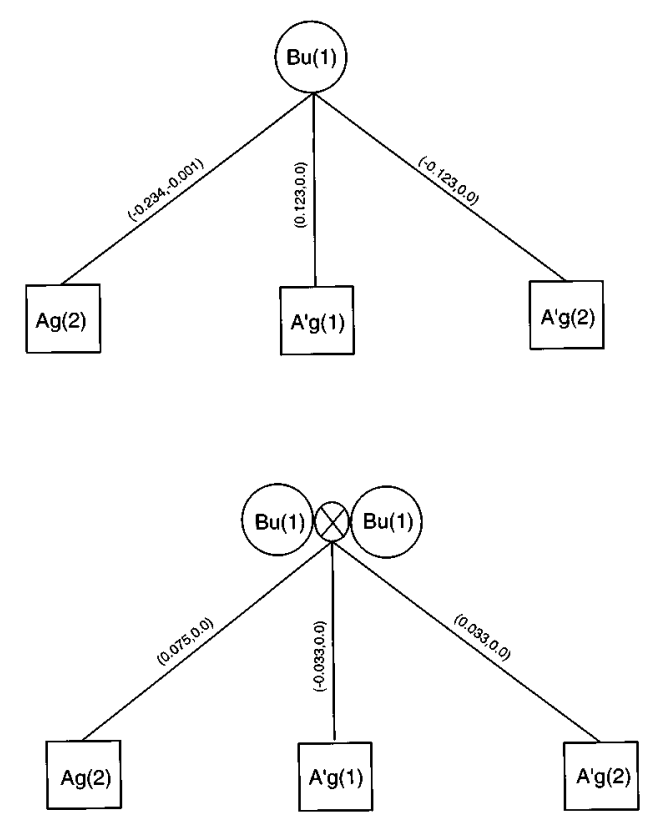

FIG. 4. Tree diagrams of first order to second order for $\omega=0.1 \mathrm{eV}$ and $\Gamma=0.1 \mathrm{eV}$. Circles, squares, and triangles represent the first-, second-, and third-order dominant oscillators. $\otimes$ stands for the coupling between two oscillators. The complex numbers along lines representing pathways are the contributions to $Q_{\nu}$ caused by corresponding pathways. 

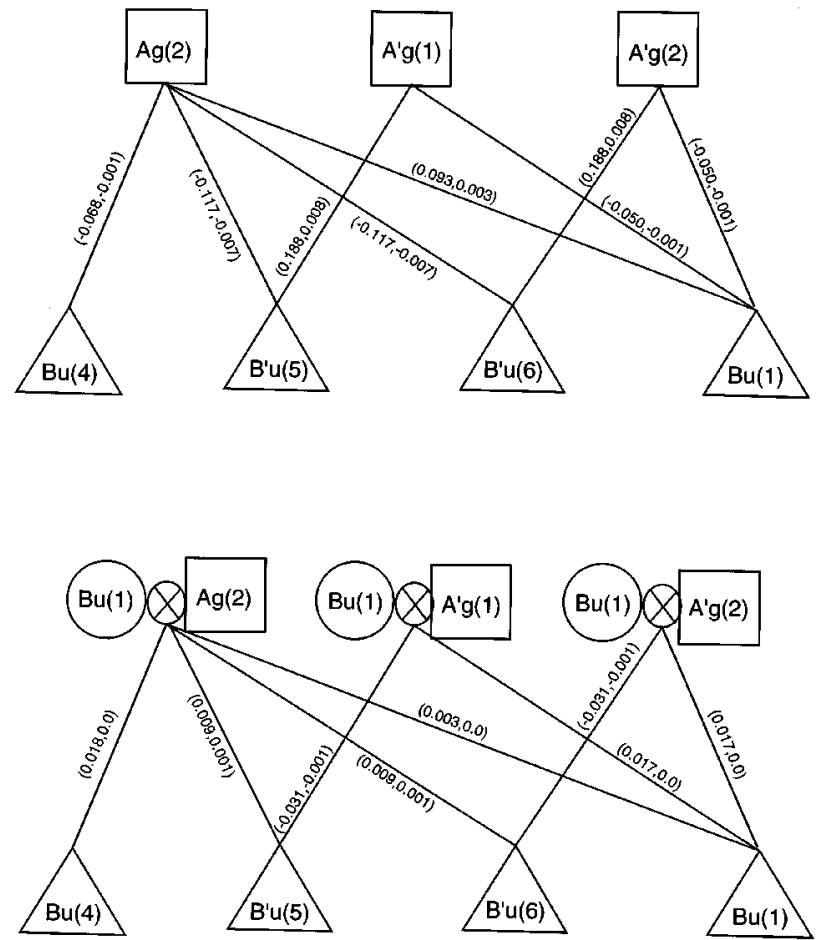

FIG. 5. Tree diagrams of second order to third order for $\omega=0.1 \mathrm{eV}$ and $\Gamma=0.1 \mathrm{eV}$.

resonances at their frequencies. In contrast, $A_{g}(2), B_{u}(1)$, and $B_{u}(4)$ contain only particle-hole components and may show up as resonances at their frequencies. Note that the couplings with the field are much stronger than the corresponding anharmonic couplings among the dominant oscillators. Note also that the contributions to various $Q_{\nu}^{(2)}$ or $Q_{\nu}^{(3)}$ from the different pathways have comparable magnitudes. However, since the dipole moment of $B_{u}(4)$ is much smaller than those of $B_{u}^{\prime}(5), B_{u}^{\prime}(6)$, and $B_{u}(1)$, this oscillator does not contribute significantly to $\gamma$. Because of cancellation between different pathways, the net contribution of $B_{u}(1)$ to $\gamma$ is relatively small as well. Therefore, the major contributions to $\gamma$ come from $B_{u}^{\prime}(5)$ and $B_{u}^{\prime}(6)$. This is illustrated in Fig. 6(a) where the high spike presents the contribution of $B_{u}^{\prime}(5)$ and $B_{u}^{\prime}(6)$ to $\gamma$. There are four dominating pathways; two positive pathways, $B_{u}(1) \rightarrow A_{g}^{\prime}(1) / A_{g}^{\prime}(2) \rightarrow B_{u}^{\prime}(5) / B_{u}^{\prime}(6)$ and two negative pathways, $B_{u}(1) \rightarrow A_{g}(2) \rightarrow B_{u}^{\prime}(5) / B_{u}^{\prime}(6)$, see upper panels of Figs. 4 and 5 . Upon grouping the positive and negative contributions, we find that the positive contribution is $(1.547,0.070) e \AA^{4} / \mathrm{V}^{3}$ and the negative is $(-0.963$, $-0.061)$ e $\AA^{4} / \mathrm{V}^{3}$. The ratio between the magnitudes of the two contributions is 1.6.

In the SOS picture, for the static limit $(\omega=0)$ there are two important channels, see Fig. 7. The first channel denoted as (I) is $1 \mathrm{Ag} \rightarrow 1 \mathrm{Bu} \rightarrow 1 \mathrm{Ag} \rightarrow 1 \mathrm{Bu} \rightarrow 1 \mathrm{Ag}$. This corresponds to the first term in Eq. (4) and its contribution is negative. The second channel denoted as (II) is $1 \mathrm{Ag} \rightarrow 1 \mathrm{Bu}$ $\rightarrow 6 \mathrm{Ag} \rightarrow 1 \mathrm{Bu} \rightarrow 1 \mathrm{Ag}$. It corresponds to the second term in Eq. (4) and its contribution is positive. For instance, at $\omega=0$, the contributions from the two channels are -1.513 e $\AA^{4} / \mathrm{V}^{3}$ and 2.920 e $\AA^{4} / \mathrm{V}^{3}$, respectively. The ratio of the magnitudes

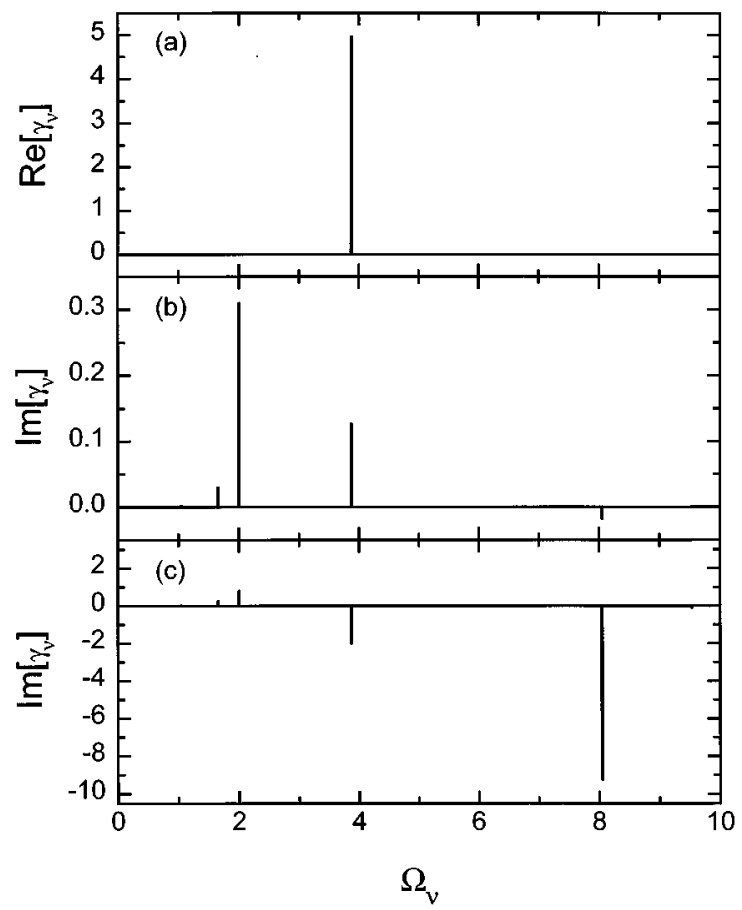

FIG. 6. $\gamma_{\nu}\left(e \AA^{4} / \mathrm{V}^{3}\right)$ vs $\Omega_{\nu}(\mathrm{eV})$ for the PPP/CEO; (a) $\operatorname{Re}\left[\gamma_{\nu}\right]$ at $\omega=0.1 \mathrm{eV}$; (b) $\operatorname{Im}\left[\gamma_{\nu}\right]$ at $1.3 \mathrm{eV}$; and (c) $\operatorname{Im}\left[\gamma_{\nu}\right]$ at $2.7 \mathrm{eV}$.

of these two contributions is 1.9. The solid lines refer to the corresponding energy levels. Since the excitations involve virtual processes, we use dashed lines to represent virtual state energy levels. Clearly, the static optical response is described adequately by a three-essential-state model.

There is some connection between the four pathways in the CEO and the two channels in the SOS. However, since there is a difference between the values of static polarizabilities among the two methods, it is difficult to establish a clear connection between the pathways and channels for the static case. This discrepancy may be accounted for by the different parameters used in the two Hamiltonians (PPP and INDO).

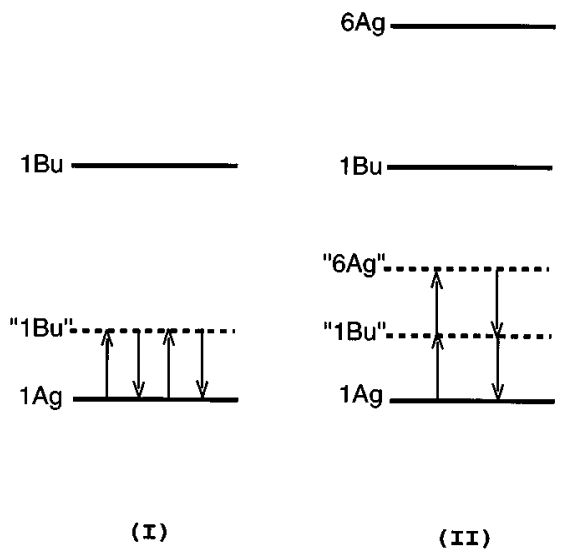

FIG. 7. Important channels (I) and (II) in the INDO/MRD-CI/SOS description. The states in quotes refer to the primary state contributing to the virtual state. 

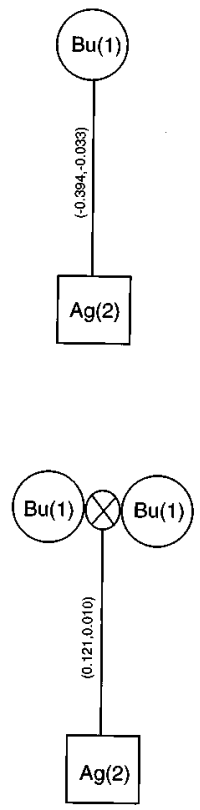

FIG. 8. Tree diagrams of first order to second order for $\omega=1.3 \mathrm{eV}$ and $\Gamma=0.1 \mathrm{eV}$.

Note that a positive pathway and a negative pathway contribute to the same oscillator $B_{u}^{\prime}(5)$ or $B_{u}^{\prime}(6)$. Thus, at third order the $Q_{\nu}$ values of $B_{u}^{\prime}(5)$ and $B_{u}^{\prime}(6)$ combine already positive and negative contributions. This is not the case in the SOS in which the negative channel (I) and the positive channel (II) contribute separately to $\gamma$.

\section{2. $3 A / 3 a$}

At resonance $3 a\left(\omega=1.3 \mathrm{eV}=0.33 \Delta_{\mathrm{CEO}}\right)$, again there is only one dominant first-order oscillator $B_{u}(1)$ (Fig. 3). More importantly, there is only one dominating oscillator in either second or third order. They are $A_{g}(2)$ and $B_{u}(1)$, respectively (Figs. 8 and 9). This is because that resonance $3 a$ corresponds an important pathway $B_{u}(1) \rightarrow A_{g}(2) \rightarrow B_{u}(1)$. The contribution of this pathway is $(-0.344,7.643) e \AA^{4} / \mathrm{V}^{3}$ compared to the total, $\gamma=(0.011,4.989)$ e $\AA^{4} / \mathrm{V}^{3}$. Like the static limit, the couplings with the external field are stronger than the anharmonic couplings among oscillators.

We performed the SOS calculation at resonance $3 A(\omega$ $=1.65 \mathrm{eV}=0.33 \Delta_{\mathrm{SOS}}$ ) and found that there is one dominant channel for $\gamma, 1 \mathrm{Ag} \rightarrow 1 \mathrm{Bu} \rightarrow 6 \mathrm{Ag} \rightarrow 1 \mathrm{Bu} \rightarrow 1 \mathrm{Ag}$, denoted as (III) in Fig. 10. Since the real energy level $1 B u$ is reached, this indicates the occurrence of a resonance, $3 \omega=E(1 B u)$ $-E(1 A g)$, where $E(m B u)$ and $E(n A g)$ are the energies of $m B u$ and $n A g$, respectively.

Clearly there is a one-to-one correspondence between pathway $\quad B_{u}(1) \rightarrow A_{g}(2) \rightarrow B_{u}(1)$ and channel (III) $1 \mathrm{Ag} \rightarrow 1 \mathrm{Bu} \rightarrow 6 \mathrm{Ag} \rightarrow 1 \mathrm{Bu} \rightarrow 1 \mathrm{Ag}$. Excited state $1 \mathrm{Bu}$ corresponds to oscillator $B_{u}(1)$, and $6 A g$ to $A_{g}(2)$.

\section{3. $(3 C, 3 D) /(3 c, 3 d)$}

At the $(3 c, 3 d)$ resonance $\left(\omega=2.7 \mathrm{eV}=0.69 \Delta_{\mathrm{CEO}}\right)$, again we find only one dominant first-order oscillator $B_{u}(1)$. In
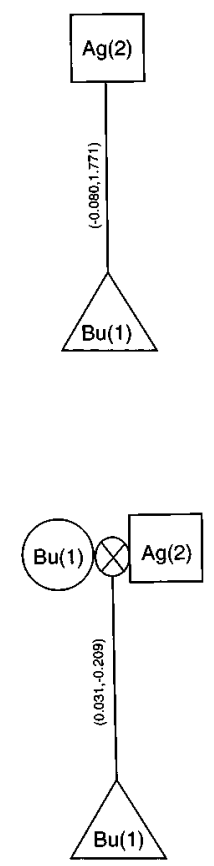

FIG. 9. Tree diagrams of second order to third order for $\omega=1.3 \mathrm{eV}$ and $\Gamma=0.1 \mathrm{eV}$.

second and third orders there are one $\left[A_{g}(2)\right]$ and two dominant oscillators $\left[B_{u}(4)\right.$ and $\left.B_{u}(1)\right]$, respectively (Figs. 11 and 12). Once again the couplings with the external field are stronger than the anharmonic couplings among oscillators. Pathway $B_{u}(1) \rightarrow A_{g}(2) \rightarrow B_{u}(4)$ dominates the amplitudes of other oscillators. However, since $B_{u}(1)$ has a much larger $d_{\nu}$, it has also a significant contribution to $\gamma$, as indicated in

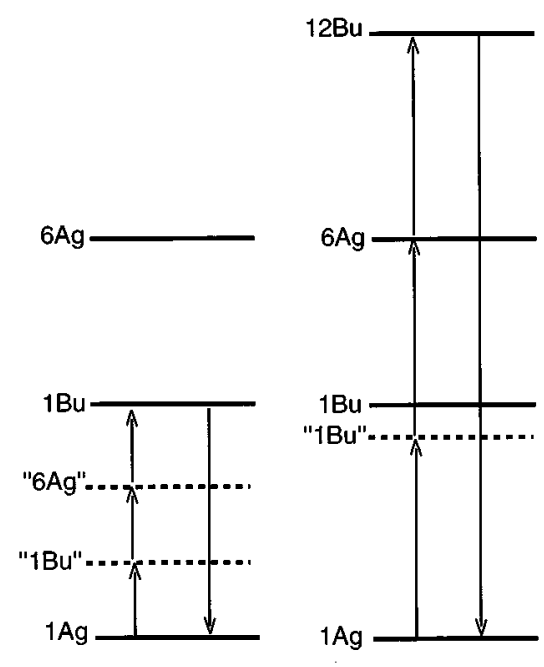

(III)

(IV)

FIG. 10. Important channels (III) and (IV) in the INDO/MRD-CI/SOS description. 

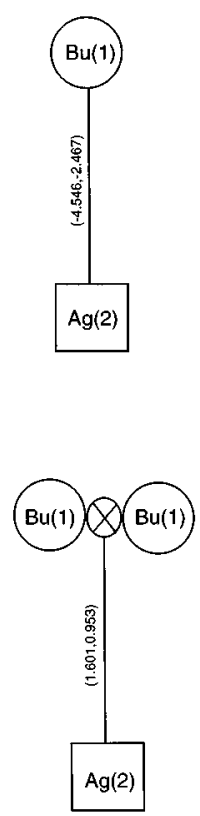

FIG. 11. Tree diagrams of first order to second order for $\omega=2.7 \mathrm{eV}$ and $\Gamma=0.1 \mathrm{eV}$.

Table III, which lists $\gamma_{\nu}$ of $B_{u}^{\prime}(5), B_{u}^{\prime}(6), B_{u}(1)$, and $B_{u}(4)$. $\gamma_{\nu}$ includes contributions to oscillator $\nu$ from all possible pathways.

We performed the SOS calculation at $(3 C, 3 D)(\omega=3.65$ $\left.\mathrm{eV}=0.74 \Delta_{\mathrm{SOS}}\right)$ and found one dominant channel for $\gamma$ : $1 \mathrm{Ag} \rightarrow 1 \mathrm{Bu} \rightarrow 6 \mathrm{Ag} \rightarrow 12 \mathrm{Bu} \rightarrow 1 \mathrm{Ag}$, which corresponds to the fourth term in Eq. (4). This process involves two resonances;
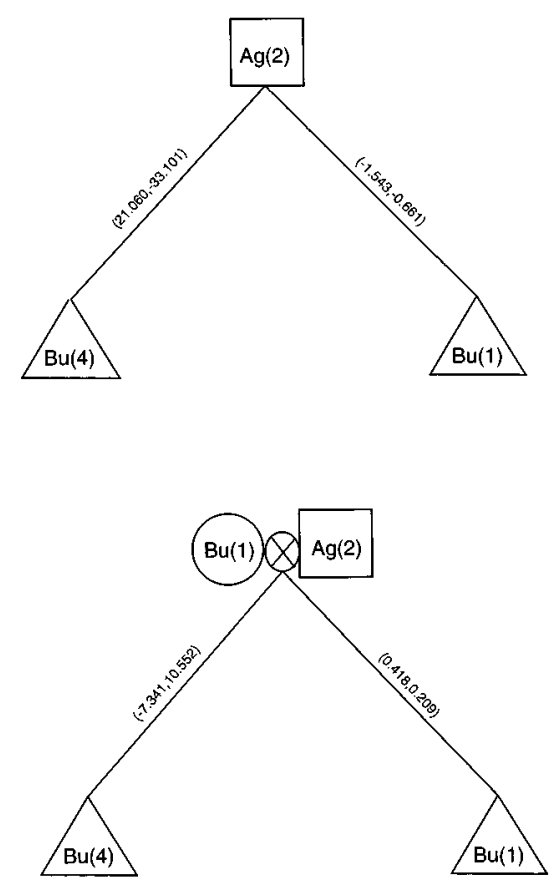

FIG. 12. Tree diagrams of second order to third order for $\omega=2.7 \mathrm{eV}$ and $\Gamma=0.1 \mathrm{eV}$.
TABLE III. The $\gamma_{\nu}$ of four dominant oscillators at different frequencies. ${ }^{\text {a }}$

\begin{tabular}{lrrr}
\hline \hline$\omega(\mathrm{eV})$ & \multicolumn{1}{c}{0.1} & \multicolumn{1}{c}{1.3} & \multicolumn{1}{c}{2.7} \\
\hline$B_{u}^{\prime}(5)$ & $(0.156,0.000)$ & $(0.204,0.009)$ & $(1.011,0.399)$ \\
$B_{u}^{\prime}(6)$ & $(0.156,0.000)$ & $(0.204,0.009)$ & $(1.011,0.399)$ \\
$B_{u}(1)$ & $(0.128,0.007)$ & $(-0.393,4.975)$ & $(-4.915,-2.008)$ \\
$B_{u}(4)$ & $(-0.019,0.000)$ & $(-0.063,-0.011)$ & $(5.629,-9.266)$ \\
Sum $^{\mathrm{b}}$ & $(0.421,0.007)$ & $(-0.048,4.982)$ & $(2.736,-10.48)$ \\
Total $^{\mathrm{c}}$ & $(0.458,0.008)$ & $(0.011,4.989)$ & $(2.787,-10.32)$ \\
\hline \hline
\end{tabular}

${ }^{\mathrm{a}} \gamma_{\nu}$ is in the units of $e \AA^{4} / \mathrm{V}^{3}$.

bum: the sum of $\gamma_{\nu}$ from $B_{u}^{\prime}(5), B_{u}^{\prime}(6), B_{u}(1)$, and $B_{u}(4)$.

'Total: the sum of $\gamma_{\nu}$ from all $B_{u}$ and $B_{u}^{\prime}$ oscillators.

(1) $3 \omega=E(12 B u)-E(1 A g), \quad$ and $\quad(2) \quad 2 \omega=E(6 A g)$ $-E(1 \mathrm{Ag})$, as indicated in Fig. 10. Obviously, a fouressential-state model is required to describe this resonance. ${ }^{17}$

Pathway $B_{u}(1) \rightarrow A_{g}(2) \rightarrow B_{u}(4)$ corresponds to channel (IV) $1 \mathrm{Ag} \rightarrow 1 \mathrm{Bu} \rightarrow 6 \mathrm{Ag} \rightarrow 12 \mathrm{Bu} \rightarrow 1 \mathrm{Ag}$, and oscillator $B_{u}(4)$ corresponds to excited state $12 B u$.

\section{Dominant oscillators/essential states}

So far we have identified seven dominant oscillators, $B_{u}^{\prime}(5), B_{u}^{\prime}(6), B_{u}(1), B_{u}(4), A_{g}^{\prime}(1), A_{g}^{\prime}(2)$, and $A_{g}(2)$. To compare the dominant oscillators and essential excited states, we project these oscillators onto HFO representation in Figs. 13 and 14. The index of HFO is defined in Ref. 3. Here are the indices of a few important Hartree-Fock oscillators; $3 \rightarrow[L, H-1], 4 \rightarrow[L, H], 7 \rightarrow[L+1, H-1], 8 \rightarrow[L+1, H]$, $22 \rightarrow[L, L+1], \quad 23 \rightarrow[H, H-1], \quad 32 \rightarrow[H, H], \quad$ and

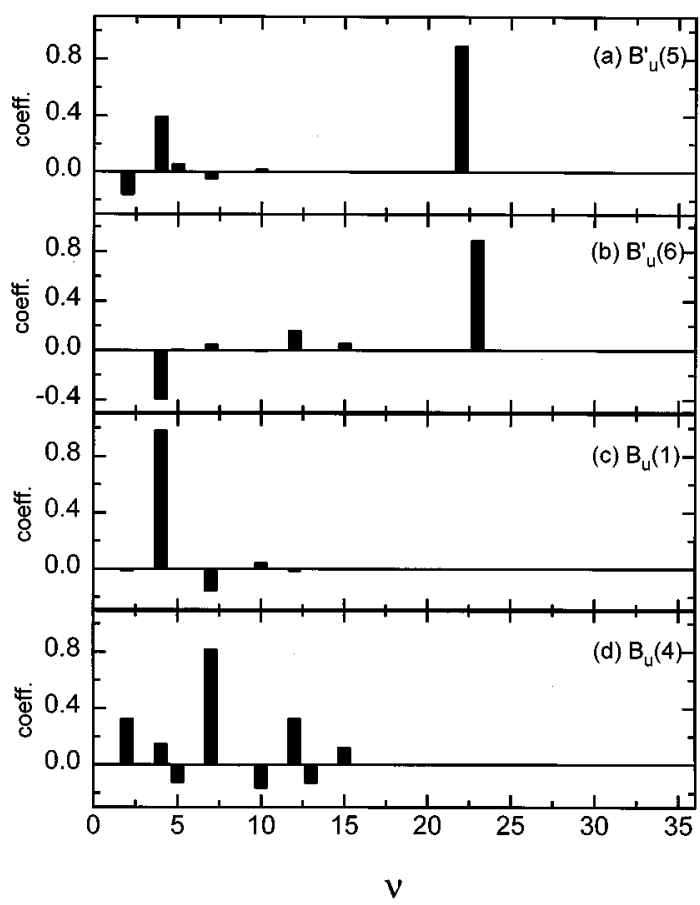

FIG. 13. HFO representation of dominant $B_{u}$ and $B_{u}^{\prime}$ oscillators. (a) $B_{u}^{\prime}(5)$; (b) $B_{u}^{\prime}(6)$; (c) $B_{u}(1)$; and (d) $B_{u}(4) . B_{u}(1)$ is mainly made of $[H, L]$, $B_{u}^{\prime}(6)$ is made mainly of $[H, H-1]$ and $[H, L], B_{u}^{\prime}(5)[L, L+1]$ and $[H, L]$ $B_{u}(4)[L+1, H-1] . \nu$ is the index of HFO 


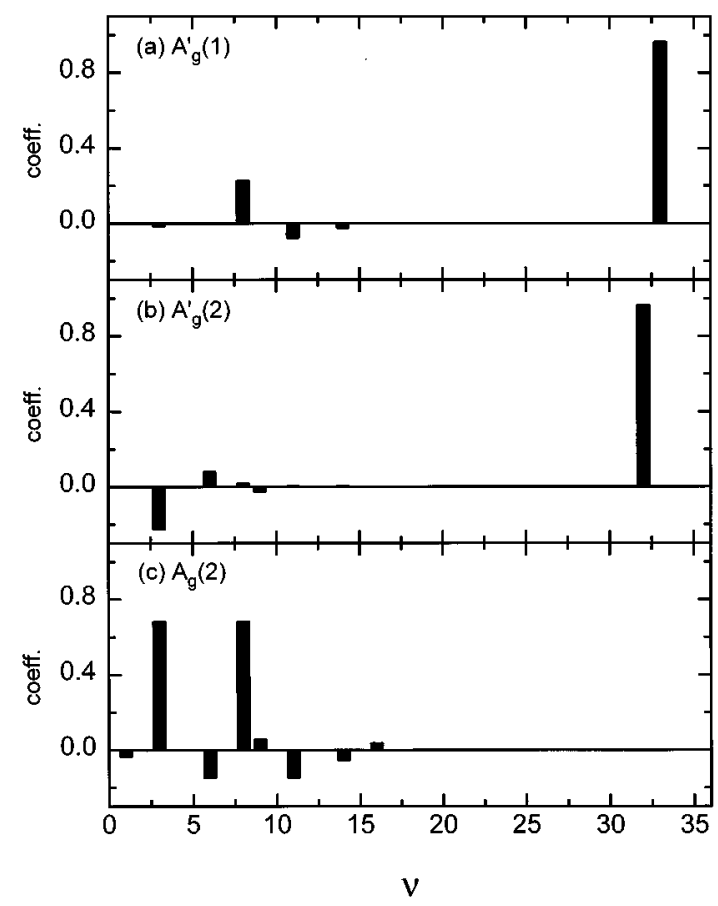

FIG. 14. HFO representation of the dominant $A_{g}$ and $A_{g}^{\prime}$ oscillators. (a) $A_{g}^{\prime}(1)$; (b) $A_{g}^{\prime}(2)$; and (c) $A_{g}(2) \cdot A_{g}^{\prime}(1)$ is mainly $[L, L], A_{g}^{\prime}(2)[H, H]$, and $A_{g}(2)[L, H-1]$ and $[L+1 ; H]$.

$33 \rightarrow[L, L]$, where $H$ and $L$ stand for HOMO and LUMO, respectively. Similar projections were plotted in Ref. 5. Obviously levels $H, H-1, L$, and $L+1$ are primarily involved in these dominant oscillators. The coefficients of various HFO components should correspond to the coefficients of the electronic configurations of excited states in the SOS description.

The essential states are listed in Table IV. $1 \mathrm{Ag}$ is basically the Hartree-Fock self-consistent ground state. $1 B u$ is approximately a single-excitation from the Hartree-Fock ground state, $H \rightarrow L$. These two are similar to the ground state and $B_{u}(1)$ in the CEO, respectively. $6 \mathrm{Ag}$ has components; $H \rightarrow L+1, H-1 \rightarrow L$, and this is similar to $A_{g}(2)$. However, it has also the double excitation $(H, H) \rightarrow(L, L)$ which differs from $A_{g}(2)$, see Fig. 14. $12 B u$ has the singleexcitation component $H-1 \rightarrow L+1$ as well as the doubleexcitation components $(H, H-1) \rightarrow(L, L)$ and $(H-1, H$ $-1) \rightarrow(L, L+1)$. The double excitation $(H-1, H$ $-1) \rightarrow(L, L+1)$ also contains the partial $H-1 \rightarrow L+1$

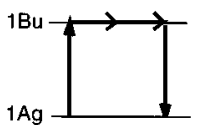

(v)

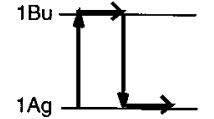

(VI)

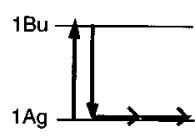

(VII)
FIG. 15. Channels corresponding to the third term of Eq. (4) in the INDO/ MRD-CI/SOS description.

excitation. This is similar to the case of the $B_{u}(4)$ oscillator, see Fig. 13.

\section{DISCUSSION}

An $A_{g}$ or $B_{u}$ oscillator corresponds exactly to a transition between the ground state and an excited state in the TDHF approximation. However, usually different approximating schemes are involved in the SOS and CEO calculations. Thus, this correspondence may only be approximate, which is the case for $B_{u}(4)$ vs $12 \mathrm{~B}$ and $A_{g}(2)$ vs $6 \mathrm{Ag}$. The $B_{u}(1)$ oscillator plays a pivotal role in the optical response of octatetraene. It is predominantly made of the transition between the frontier molecular orbitals (HOMO and LUMO) and is the only dominant oscillator in first order. Although at the static, $3 a$ and $3 c / 3 d$ limits other oscillators $B_{u}(4)$, $B_{u}^{\prime}(5)$, and $B_{u}^{\prime}(6)$ are involved in third order processes, $B_{u}(1)$ always has an important contribution.

The dominant oscillators, $A_{g}^{\prime}(1), A_{g}^{\prime}(2), B_{u}^{\prime}(5)$, and $B_{u}^{\prime}(6)$ are mainly made of particle-particle or hole-hole components. The frequencies of $A_{g}^{\prime}$ and $B_{u}^{\prime}$ oscillators are simply the differences between two occupied or unoccupied Hartree-Fock molecular orbitals. When the two orbitals are different, these oscillators correspond approximately to the transitions between two excited states (i.e., intraband excitations). Since the resonant frequencies are determined by the energy differences between the excited states and ground state and not by the energy differences among the excited states, these oscillators do not lead to resonances at their frequencies. $A_{g}^{\prime}(1)$ and $A_{g}^{\prime}(2)$ are $H \rightarrow H$ and $L \rightarrow L$, respectively. They constitute major parts of the second-order optical processes and contribute strongly to the emergence of third-order response at the static limit. They associate with the third term in Eq. (4), which may be expressed by the channels propagating along the ground state $1 \mathrm{Ag}$ and excited state $1 B u$ respectively, as depicted in Fig. 15. By symmetry,

TABLE IV. Components of the ground and excited states. ${ }^{\mathrm{a}}$

\begin{tabular}{lcccccccc}
\hline \hline & hfgs $^{\mathrm{b}}$ & $h \rightarrow l$ & $h \rightarrow l^{\prime}$ & $h^{\prime} \rightarrow l$ & $h^{\prime} \rightarrow l^{\prime}$ & $h h \rightarrow l l$ & $h h^{\prime} \rightarrow l l$ & $h^{\prime} h^{\prime} \rightarrow l l^{\prime}$ \\
\hline $\begin{array}{l}1 \mathrm{Ag} \\
1 \mathrm{Bu}\end{array}$ & 0.93 & & & & & & & \\
$6 \mathrm{Ag}$ & 0.15 & 0.92 & & 0.35 & 0.59 & -0.15 & & \\
$12 \mathrm{Bu}$ & & & & & -0.21 & & 0.51 & 0.34 \\
\hline \hline
\end{tabular}

${ }^{a} h: H, l: L, h^{\prime}: H-1$, and $l^{\prime}: L+1$.

${ }^{\mathrm{b}} \mathrm{hfgs}$, Hartree-Fock ground state. 
$A_{g}^{\prime}(1)$ and $A_{g}^{\prime}(2)$ have zero dipole moments and do not appear in third order. However, when the symmetry is broken, the situation may change. For instance, in the case of DANS, these two oscillators appear in third order and contribute substantially to the value of $\gamma \cdot{ }^{13}$

At resonance frequencies, there is usually only one important channel in the SOS picture, and correspondingly, one dominating pathway in the CEO picture in terms of amplitude. Therefore, there is a one-to-one correspondence between the dominating pathway in the CEO and the important channel in the SOS. However, since the dipole moments $d_{\nu}$ of other oscillators may be much larger than those of oscillators related with the dominating pathway, there may be significant contributions to the polarizabilities from other pathways. This is illustrated clearly in Fig. 12.

For the static or near static case, the four dominating pathways (two positive and two negative) in the CEO picture seem to correspond to the two important channels in the SOS formalism. The exact nature of this correspondence is not clear since there are some differences between the PPP and INDO Hamiltonians and there is discrepancy in the resulting values of the static second hyperpolarizability $\gamma$. At third order, the oscillators $B_{u}(5)$ and $B_{u}(6)$ have combined automatically the contributions from the positive and the negative channels. This is different from the case in the SOS formalism where the contributions from the positive and negative channels add only at the very end. This constitutes a major advantage of the CEO formalism.

Another advantage of the CEO formalism is that different sources of nonlinearities can be easily identified. ${ }^{3} \mathrm{We}$ noticed that the contributions to $Q_{\nu}$ of each dominant oscillator from the coupling with the external field is much stronger than those induced by the couplings among oscillators. The couplings with the external field do not depend on electron-electron interaction explicitly. This seems to indicate that the Hückel model is appropriate for describing the THG spectra of polyenes. Further investigation is warranted to unveil the origin and implications of this observation.

The CEO formalism is based on the TDHF procedure. ${ }^{2,3}$ The excitations in the TDHF formalism not only include single electron excitations explicitly but also double, triple and multiple excitations implicitly. ${ }^{26}$ On the other hand, in the SOS calculations, double, triple, and other multiple excitations have to be written down. This increases the size of configuration interaction $(\mathrm{CI})$ calculation rapidly. For instance, to have a good description of $12 \mathrm{Bu}$ state, the INDO/ $\mathrm{MRD}-\mathrm{CI} / \mathrm{SOS}$ calculation has to take into account single-, double-, and even triple-excitations, see Table IV. The implicit inclusion of multiexcitations in the PPP/CEO formalism greatly reduces the computational effort, yet retains some essential physical processes automatically and thus leads to good agreement with considerably more intensive INDO/MRD-CI/SOS calculations.

\section{ACKNOWLEDGMENTS}

The work in Rochester has been supported by the Air Force Office of Scientific Research, the National Science
Foundation, and the NSF Center for Photoinduced Charge Transfer. The work in Mons has been partly supported by the Belgian Government "Services Fédéraux des Affaires Scientifiques, Techniques et Culturelles" (Pôle d'Attraction Interuniversitaire No. 16: Chimie Supramoléculaire et Catalyse), FNRS/FRFC, and an IBM Academic Joint Study. D.B. is Chargé de Recherches of the Belgian National Fund for Scientific Research (FNRS) and is grateful to FNRS and the NSF Center for Photoinduced Charge Transfer for support during his stay Rochester.

${ }^{1}$ S. Mukamel and H. X. Wang, Phys. Rev. Lett. 69, 65 (1992).

${ }^{2}$ A. Takahashi and S. Mukamel, J. Chem. Phys. 100, 2366 (1994).

${ }^{3}$ G. Chen and S. Mukamel, J. Am. Chem. Soc. 117, 4945 (1995).

${ }^{4}$ (a) S. Mukamel, A. Takahashi, H. X. Wang, and G. Chen, Science 266, 251 (1994); (b) G. Chen, A. Takahashi, and S. Mukamel, Proc. SPIE 2143, 142 (1994).

${ }^{5}$ G. Chen and S. Mukamel, Chem. Phys. Lett. 240, 296 (1995).

${ }^{6}$ (a) G. Chen and S. Mukamel, J. Chem. Phys. 103, 9355 (1995); (b) J. Phys. Chem. (submitted).

${ }^{7}$ B. J. Orr and J. F. Ward, Mol. Phys. 20, 513 (1971).

${ }^{8}$ D. S. Chemla and J. Zyss, Nonlinear Optical Properties of Organic Molecules and Crystals (Academic, New York, 1987).

${ }^{9}$ A. F. Garito, J. R. Heflin, K. Y. Wong, and O. Zamani-Khamiri, in Organic Materials for Nonlinear Optics, edited by R. A. Hann and D. Bloor (Royal Society of Chemistry, London, 1989), p. 16.

${ }^{10}$ (a) S. Etemad and Z. G. Soos, in Spectroscopy of Advanced Materials, edited by R. J. H. Clark and R. E. Hester (Wiley, New York, 1991), p. 87; (b) Z. G. Soos, S. Ramasesha, D. S. Galvao, and S. Etemad, Phys. Rev. B 47, $1742(1993)$

${ }^{11}$ J. M. André, J. Delhalle, and J. L. Brédas, Quantum Chemistry Aided Design of Organic Polymers. An Introduction to the Quantum Chemistry of Polymers and its Applications (World Scientific, Singapore, 1991).

${ }^{12}$ (a) W. J. Buma, B. E. Kohler, and T. A. Shaler, J. Chem. Phys. 96, 399 (1992); (b) B. E. Kohler, Chem. Rev. 93, 41 (1994).

${ }^{13}$ D. Beljonne, J. L. Brédas, G. Chen, and S. Mukamel, Chem. Phys. (in press).

${ }^{14}$ (a) D. Beljonne, Z. Shuai, and J. L. Brédas, J. Chem. Phys. 98, 8819 (1993); (b) Z. Shuai, D. Beljonne, and J. L. Brédas, J. Chem. Phys. 97, $1132(1992)$

${ }^{15}$ (a) D. Beljonne and J. L. Brédas, J. Opt. Soc. Am. B 11, 1380 (1994); (b) D. Beljonne, J. L. Brédas, M. Cha, W. E. Torruellas, G. I. Stegeman, J. W. Hofstraat, W. H. G. Horsthuis, and G. R. Möhlmann, J. Chem. Phys. 103, 7834 (1995)

${ }^{16}$ B. M. Pierce, J. Chem. Phys. 91, 791 (1989).

${ }^{17}$ (a) D. Guo, S. Mazumdar, S. N. Dixit, F. Kajzar, Y. Kawabe, and P. Peyghambrian, Phys. Rev. B 48, 1433 (1993); (b) D. Guo, S. Mazumdar, G. I. Stegeman, M. Cha, D. Neher, S. Aramaki, W. E. Torruellas, and R. Zanoni, Mater. Res. Soc. Symp. Proc. 247, 151 (1992).

${ }^{18}$ P. Ring and P. Schuck, The Nuclear Many-Body Problem (Springer, New York, 1980).

${ }^{19}$ D. J. Rowe, Rev. Mod. Phys. 40, 153 (1968)

${ }^{20}$ H. Sekino and R. J. Bartlett, J. Chem. Phys. 85, 976 (1986).

${ }^{21}$ (a) L. A. Heimbrook, J. E. Kenny, B. E. Kohler, and G. W. Scott, J. Chem. Phys. 75, 4338 (1981); (b) D. G. Leopold, V. Vaida, and M. F. Granville, ibid. 81, 4210 (1984).

${ }^{22}$ In Ref. 5, $A_{g}$ are for all the gerade $(g)$ oscillators and $B_{u}$ for all the ungerade $(u)$ oscillators.

${ }^{23}$ J. Ridley and M. Zerner, Theor. Chim. Acta 32, 111 (1973).

${ }^{24}$ R. J. Buenker and S. D. Peyerimhoff, Theor. Chim. Acta 35, 33 (1974).

${ }^{25}$ M. J. S. Dewar, E. G. Zoebisch, E. F. Healy, and J. J. P. Stewart, J. Am. Chem. Soc. 107, 3902 (1985).

${ }^{26}$ A. L. Fetter and J. D. Walecka, Quantum Theory of Many-Particle Systems (McGraw-Hill, New York, 1971). 